\title{
Anabases
}

ANABASES Traditions et réceptions de l'Antiquité

$23 \mid 2016$

Varia

\section{Fontes Inediti Numismaticae Antiquae : présentation succincte d'un nouveau projet antiquaire}

\section{François de Callataÿ}

\section{(2) OpenEdition}

\section{Journals}

Édition électronique

URL : http://journals.openedition.org/anabases/5640

DOI : 10.4000/anabases.5640

ISSN : 2256-9421

Éditeur

E.R.A.S.M.E.

\section{Édition imprimée}

Date de publication : 2 mai 2016

Pagination : 163-168

ISSN : 1774-4296

\section{Référence électronique}

François de Callatäy, «Fontes Inediti Numismaticae Antiquae : présentation succincte d'un nouveau projet antiquaire », Anabases [En ligne], 23 | 2016, mis en ligne le 02 mai 2019, consulté le 20 octobre 2019. URL : http://journals.openedition.org/anabases/5640 ; DOI : 10.4000/anabases.5640

(c) Anabases 
Anabases 23 (2016), р. г63-г68.

\section{Fontes Inediti Numismaticae Antiquae: présentation succincte d'un nouveau projet antiquaire}

François de Callatä̈

e projet Fontes Inediti Numismaticae Antiquae (FINA en acronyme) a été lancé de façon informelle en novembre 2oII quand un groupe de collègues et d'amis —s'est retrouvé à la Berlin-Brandenburgische Akademie der Wissenschaften pour le colloque international Translatio Nummorum. Römische Kaiser in der Renaissance (I6-I8 novembre 20II). Ce premier cercle berlinois comprenait John Cunnally, Ursula Kampmann, Federica Missere Fontana, Ulrike Peter, Bernhard Woytek et moi-même, ainsi que Michael Alram et Bernhard Weisser qui nous ont rejoints presque immédiatement.

Nous avons alors procédé à un double constat commun: d'abord, celui d'un intérêt général toujours grandissant pour l'historiographie et l'antiquarianisme, ensuite et surtout, celui d'une prise de conscience que, jusqu'ici, les études numismatiques de ce type se sont presqu'exclusivement fondées sur les ouvrages imprimés, lesquels - aussi nombreux soient-ils (voir à ce sujet les impressionnants catalogues établis par Yvette et Christian Dekesel) - ne forment que la pointe de l'iceberg.

Au-delà des livres imprimés, qui ont fondamentalement façonné le développement de nos connaissances, il existe d'autres types de sources, généralement ignorées par ceux qui ont tenté d'écrire l'histoire de la numismatique antique. Or celles-ci sont absolument nécessaires si l'on veut approcher ce qu'on appelait alors la République des médailles. Les archives sont pleines de manuscrits qui n'ont jamais été publiés, en particulier ceux qui étaient trop techniques ou trop coûteux pour être imprimés.

Les bibliothèques possèdent de nombreux exemplaires de livres richement annotés par des érudits célèbres. Ainsi, possédons-nous de nombreux exemplaires 
annotés de l'Imperatorum romanorum numismata, l'ouvrage de référence sur les monnaies impériales romaines publié par Adolph Occo en i579. L'un d'entre eux, conservé à Bologne, est passé entre les mains de quatre antiquaires célèbres qui l'ont tous enrichi de commentaires: Dionigi Ottaviano Sada, Lodovico Compagni, Leonardo Agostini et Giovanni Pietro Bellori. Mais on trouve, toujours à Bologne, un exemplaire annoté par Fulvio Orsini tandis que Cambridge possède l'exemplaire d'Abraham Ortelius et Carpentras, bien entendu, celui de Peiresc, annoté ultérieurement aussi par Louis Thomassin de Mazaugues.

De toutes les sources potentielles pour reconstruire ce que fut la République des Médailles (et il faudrait encore citer les testaments, les carnets de voyage, les journaux intimes, etc.), la correspondance forme très probablement le type de documentation le plus vaste et dont il y a le plus à attendre. L'étude de ces correspondances, d'une ampleur considérable, non seulement colore le récit désincarné des livres en donnant à voir le chatoiement de ce qui fait la condition humaine (dont ses vilénies comme la jalousie, la passion, l'ambition, la fatuité et la cupidité), mais elle rend compte surtout d'un réseau beaucoup plus vaste de personnes connectées entre elles que ce qui se peut glaner dans les livres: non plus quelques dizaines d'érudits mais des centaines de curiosi, plus ou moins lettrés, plus ou moins collectionneurs. Ce monde vibrant, pour lequel l'authenticité et la rareté des pièces représentaient une connaissance nécessaire à l'établissement du prix, fait qui plus est état d'un connoisseurship souvent supérieur à ce qui se déduit des ouvrages imprimés dont il nous donne à voir une réalité plus vivante, plus large et mieux informée. Dans Testimoni parlanti. Le monete antiche a Roma tra Cinquecento e Seicento, publié à Rome en 2009, Federica Missere Fontana, qui exploite des centaines de lettres, offre une très belle illustration de ce que les sources non imprimées ont à offrir à ce propos.

Le but de Fina est donc de réunir, de lire, d'étudier et de publier les sources non imprimées relatives à la numismatique antique, avant tout gréco-romaine. S'agissant des sources imprimées, Christian Dekesel, qui mérite bien d'être dénommé le père moderne (l'ancien est Johann Gottfried Lipsius, I756-I820), de la bibliographie numismatique antiquaire, n'a pas craint d'écrire:

The enormous volume of numismatic publications prior to I8or is largely unknown to numismatists of the twentieth century, with the existing bibliographies containing incomplete information about most of them. By researching these early numismatic publications one becomes aware of their importance for the evolution and progression of numismatic knowledge. This brings us to the Axiom of Informational Relevance as our prime motivation. The basis for this axiom is that all publications concerning a certain subject, published at any time in the past, at any place and in any language, can have some relevance towards the actual scientific study of that subject today. What somebody published about a certain subject three hundred years ago can be as relevant for that particular subject as that which somebody else has discovered 
about it last week with the advantage of the most modern technological equipment. It is totally wrong to assume that because a printed source is old, it is unreliable, irrelevant, meaningless or outdated. From a pure historical point of view there is probably nobody who will oppose this statement, but from a scientific approach there is always the possibility that modern scientists or researchers may have overlooked an essential aspect of a problem which somebody else in the past has noticed. Some future scientist can suddenly realise that a past statement, which was looked upon by his predecessors as irrelevant or even wrong, can be of importance for further scientific evolution within a specific subject. Without this axiom any attempt to establish a comprehensive bibliography on a certain subject would be a pure waste of time, or reduced to a pastime for some alienated researcher who can only fulfil his deepest aspirations by doing something in which nobody is interested!

On ajoutera à cette déclaration très positive que les correspondances sont par excellence le lieu des querelles, c'est-à-dire celui des réseaux, et que ces correspondances permettent mieux qu'aucune autre source documentaire sans doute, d'étudier l'histoire des erreurs: sur quelles bases se sont-elles créées et comment se sont-elles diffusées? C'est toute la question de la production et de la transmission du savoir qui est ici exposée, à travers le kaléidoscope des positions personnelles.

Chronologiquement, nous avons décidé de prendre i8oo comme date butoir et cela pour deux raisons: d'abord parce que cette date correspond presque à celle du décès de Joseph Eckhel (I737-I798), passé à la postérité comme «le père de la numismatique moderne "; ensuite parce que, à de rares exceptions (comme Celestino Cavedoni à Modène ou Ludwig Müller à Copenhague), les décennies du milieu du $\mathrm{XIX}^{\mathrm{e}} \mathrm{s}$. sont caractérisées par un déclin sensible de la littérature numismatique qui va constituer comme un fossé pour les générations futures, désormais amnésiques de la tradition antérieure.

Lancé fin 20II, le projet FINA n'a pas tardé à recevoir de prestigieux patronages. Nous nous sommes d'abord tournés, Michael Alram et moi, vers nos propres académies nationales, l'Österreichische Akademie der Wissenschaften et l'Académie royale de Belgique, qui nous ont immédiatement accordé leur patronage. En avril 20ı3, lors de son assemblée générale tenue à Mayence, l'Union académique internationale a officiellement accueilli le projet finA comme la $83^{\mathrm{e}}$ entreprise dont elle soutient les travaux. Ensuite, nous avons cherché et obtenu le soutien supplémentaire de l'Académie des inscriptions et belles-lettres à Paris, de la Berlin-Brandenburgische Akademie der Wissenschaften à Berlin et de l'Unione Accademica Nazionale à Rome. Par ailleurs, plus de 35 collègues de différents pays et continents ont manifesté leur souhait de rejoindre FinA.

Dès 2012, Bernhard Woytek avait introduit auprès du Fonds autrichien pour la science (FWF) un projet, placé sous l'égide de FINA, et visant à publier la correspondance passive reçue par Joseph Eckhel et aujourd'hui conservée au cabinet des médailles du Kunsthistorisches Museum de Vienne. Le financement de ce projet 
(Joseph Eckhel [I737-I798] and his Numismatic Network - projet $\mathrm{n}^{\circ} \mathrm{P}_{25282}$ ) a permis d'engager Daniela Williams pour trois ans et de tenir une conférence internationale du 27 au 3omai 2015 réunissant une vingtaine de collègues, dont plusieurs spécialistes renommés de l'histoire des idées à l'époque des Lumières (Ars Critica Numaria. Joseph Eckhel and the development of numismatic method).

En dépit de ces belles réalisations autrichiennes, le projet fiNa est plus généralement toujours dans les limbes: l'enthousiasme est certainement là, mais pas le financement et des décisions importantes doivent encore être prises s'agissant des normes éditoriales communes qu'il serait souhaitable d'adopter.

Toutefois, sans attendre d'être financé pour ce faire,j'ai, de façon plus ou moins frénétique et avec l'aide précieuse à partir d'un certain point de Guy Meyer, entrepris de créer ce que - faute de mieux - nous appelons «le Grand Document». Il s'agit d'un outil de travail qui réunit tout ce que nous avons trouvé relatif aux sources numismatiques non imprimées avant I8oo. Précédé d'une note aux utilisateurs et ponctué par un index des noms et une bibliographie, ce Grand Document est inégal, incomplet et non expurgé de bien des coquilles, mais - même comme tel -il représente déjà une mine d'or à partir de laquelle on peut extraire des informations inédites sur à peu près tous les sujets et toutes les personnalités qui firent partie de la République des Médailles. Pour la correspondance, il contient tout ce qu'il a semblé pertinent d'inclure, à savoir a) la correspondance déjà publiée (par exemple celle de Gisbert Cuper ou d'Apostolo Zeno); b) les lettres citées dans la littérature secondaire, à commencer par les nombreux articles de Federica Missere Fontana, mais aussi - et de plus en plus - c) les lettres inédites numérisées et maintenant disponibles sur Internet à travers divers sites Web, à commencer par Gallica, pionnier et modèle du genre.

Dans son état actuel (septembre 2or5), le Grand Document se présente sous la forme d'un gros fichier Word, approchant les 2,5 millions de caractères. Il reprend 79 inventaires de collections, 75 manuscrits inédits, et - en tout ou en partie - le contenu d'environ 2500 lettres écrit par plus de 300 auteurs pour un total de 450 correspondants (il est cependant à penser que ces 2500 lettres ne représentent pas même un tiers de ce qui est potentiellement disponible).

En tête des correspondances passives, on trouve Bernard de Montfaucon, peu porté lui-même à l'étude des monnaies mais dont le succès phénoménal de $L$ 'Antiquité expliquée avait entraîné pour lui une correspondance avec des centaines de ses contemporains, dont 47 l'entretiennent notamment de numismatique. Ces derniers sont essentiellement des personnages sans aucune incidence sur l'histoire de la science des monnaies, mais dont les lettres peuvent à l'occasion se révéler très éclairantes. Pour n'en donner qu'un exemple, David Auerbach, un libraire de Leipzig, lui explique ainsi en mars I73ı qu'il a envoyé à Paris les trois volumes du Thesaurus Brandenburgicus publiés par Lorenz Beger dans les années i6g6-ı7o2, mais que - comme d'habitude, écrit-il - ces Messieurs les libraires de Paris n'en 
ont fait aucun cas. Il implore dès lors Montfaucon, pour le salut de la veuve de Beger, de recommander cet ouvrage aux amateurs parisiens. Le point intéressant n'est pas tant d'apprendre que Lorenz Beger, ecclésiastique protestant, était marié ou qu'il avait publié son ouvrage en partie au moins à compte d'auteur, mais que son Thesaurus Brandenburgicus, une référence majeure de nos jours pour toute personne intéressée à la réception de l'antiquité, n’était toujours pas reçu à Paris, trois décennies après sa mort.

Si l'on s'intéresse aux numismates pour lesquels nous disposons du plus grand nombre de lettres écrites, on voit que les Italiens sont très bien représentés: ceux du second tiers du XvII ${ }^{\mathrm{e}}$ s. comme Francesco Gottifredi, Ottavio Falconieri et Leonardo Agostini, ou Apostolo Zeno une génération plus tard. Le tournant des XVII $^{\mathrm{e}}$ et $\mathrm{XVIII}^{\mathrm{e}} \mathrm{s}$. est aussi le moment qui voit le Hollandais Gisbert Cuper, véritable Peiresc de son temps, créer son énorme volume de correspondances, aujourd'hui conservées à La Haye, en particulier avec Antoine Galland.

Si l'on considère les périodes au cours desquelles ces quelque 25 oo lettres ont été écrites, on observe des évolutions aux contrastes parfois violents. Avec plus de $70 \%$ de toutes les lettres, la période qui va de i640 à I740 apparaît comme le siècle d'or pour la correspondance numismatique. À l'inverse, les années i740 et r75o forment le nadir de ce mouvement.

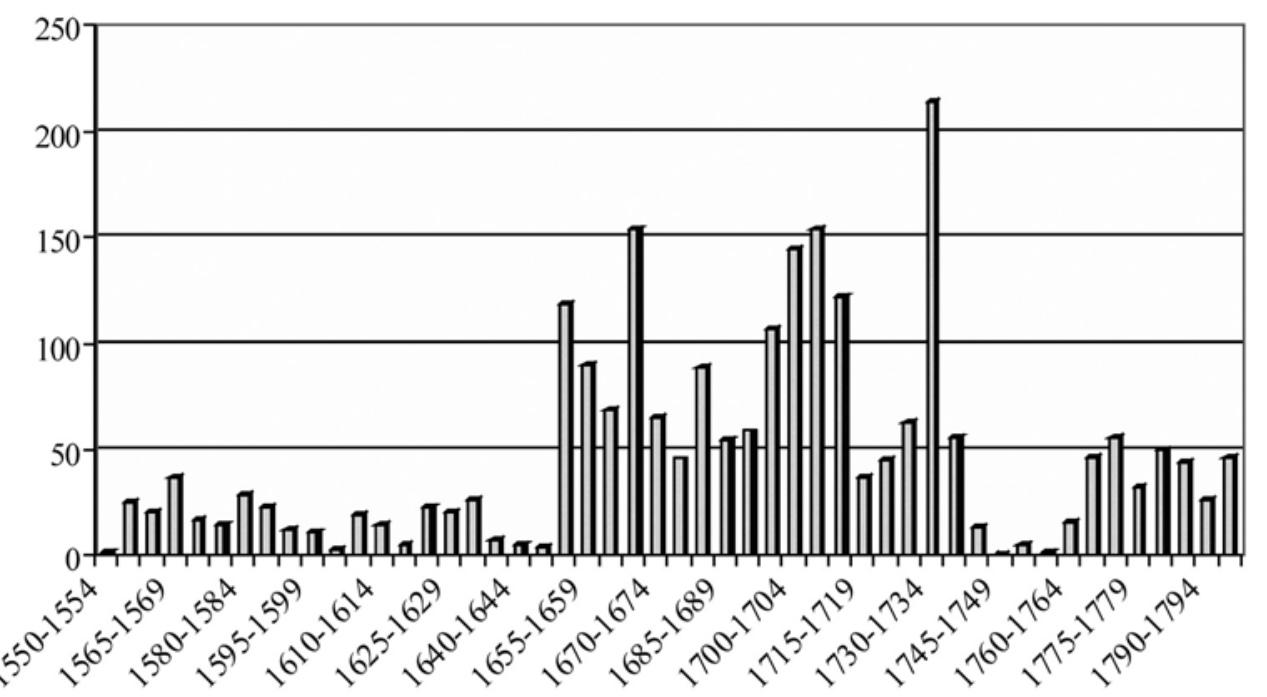

Graphique I. Nombre de lettres enregistrées par périodes de cinq ans dans le Grand Document (I550-180o).

Si l'on prend en compte l'évolution générale de cinq en cinq ans, le phénomène le plus remarquable est sans doute l'augmentation vertigineuse (de i à 3o) intervenue entre les années i645-ı649 (4 lettres seulement) et les années i65o-r654 (pas 
moins de iı8 lettres). À première vue, et même si la plupart des lettres datées des années i65o ont été échangées en Italie, il est très tentant de faire le lien entre cette floraison épistolaire et la fin de la Guerre de Trente Ans qui a dévasté l'Europe de ı6ı 8 à ı648. D'autre part, l'extrême rareté des lettres pour les années I745 à I759 s'explique le mieux, ainsi que l'ont démontré pour la France Antoine Schnapper et Jean-Baptiste Giard, par une désaffection générale pour les monnaies antiques. On trouve dans la correspondance du vénitien Apostolo Zeno de précieux témoignages à ce sujet. Ainsi écrit-il en août I735 à Gianfrancesco Baldini : “Le medaglie oggidì, e più in Venezia che in Roma, sono una mercantazia quasi del tutto fallita, non essendoci chi ne faccia gran caso, perchè non vi è chi ne abbia la vera intelligenza e il buon gusto. s Il s'était plaint de la même manière en janvier I734 à Giandomenico Bertoli: “Qui pochi sono i dilettanti, e molto meno gl'intendenti. Questo studio, che quasi in questa città (nb: Venise) ha cominciato, va a poco a poco mancando.» Une des raisons de cette désaffection est qu'il était devenu désormais très difficile de trouver des pièces de monnaie anciennes, ce qui avait poussé plusieurs, dont Zeno, à commencer à réunir les monnaies modernes. Zeno s'explique sur la raison de cette difficulté dans une lettre à Baldini datée de mai ı736: “Mi è stato di molto piacere l'intendere dalla lettera di V.P.Reverendissima, che le medaglie d'oro del museo Gualtieri sieno passate in mano del nostro Signor Cardinal Quirini, e però sieno rimaste in Italia, nè abbiano corsa la sorte di tante altre simili rarità, di andare di là dai monti, e dai mari ad arricchire le straniere nazioni, che pur troppo delle nostre spoglie sen vanno belle e superbe.» On voit par ces quelques extraits tirés de la correspondance de Zeno ce que les échanges épistolaires ont à offrir et que l'on chercherait vainement dans les livres.

Guy Meyer et moi-même sommes tout disposés à communiquer le Grand Document à celles et ceux qui en feraient la demande (callatay@kbr.be) et, bien entendu, plus heureux encore de pouvoir remercier celles et ceux qui nous feraient parvenir des accroissements à celui-ci.

\section{François de Callataÿ}

Bibliothèque royale de Belgique École pratique des Hautes Études Université libre de Bruxelles callatay@kbr.be 